\title{
Velocardiofacial syndrome - a syndrome with many faces
}

M Lombard, MB ChB, BA, MFamMed

J L Roos, MB ChB, MMed (Psych), MD, FCPsych (SA)

Department of Psychiatry, University of Pretoria, and Weskoppies Hospital, Pretoria

Among the many features with which velocardiofacial syndrome (VCFS) may present, we review in this paper the literature on working memory deficits, dysmorphic features, co-morbid obsessive-compulsive disorder, early deviant behaviour, functional impairment, and early age of onset of schizophrenia. These features are considered in a case series of 5 patients, and in addition we report on uncommon features. We highlight the importance of appropriate genetic counselling and need for preventive treatments in the future. Considering that covert symptoms in VCFS may easily be overlooked, we call for clinical vigilance in detecting VCFS among patients suffering from schizophrenia using the Bassett and Chow criteria, because the diagnosis of VCFS is of importance in determining prognosis and providing appropriate genetic counselling.

MERGEFIELD backgroundVelocardiofacial syndrome (VCFS), also known as DiGeorge or Shprintzen syndrome, is associated with small deletions of chromosome 22q11. It was first described by Shprintzen et al. and has an estimated prevalence of 1 in 5950 births, making it the second most common genetic syndrome after Down syndrome. ${ }^{1,2}$ It has been shown that patients with a hemizygous deletion of the q11 band of chromosome 22 are at a high risk of serious psychiatric illnesses, schizophrenia being the most common of these. ${ }^{3.8}$ They have a 29 times higher risk than the general population of developing schizophrenia and 3 times the risk of having a first-degree relative with schizophrenia. ${ }^{8.9}$ The familial pattern of schizophrenia has been recognised for a long time., ${ }^{1,0-12}$ The $22 q 11$ deletion syndrome (DS) occurs most frequently as a sporadic mutation in schizophrenia, but inheritance plays a role in $10 \%$ of cases.

According to Bassett and Chow, patients with two or more of the following groups of screening criteria are at an increased risk for 22q11 DS:

- hypernasal speech, history of speech therapy, velopharyngeal incompetence, cleft palate

- characteristic facial features - long narrow face, small low-set ears, a small mouth and retruded chin

- learning difficulties, history of special education

- congenital heart disease - e.g. ventrical septal defect (VSD) and tetralogy of Fallot

- other significant congenital abnormalities - e.g. club feet, polydactyly, and hypospadias

- history of hypocalcaemia or hypoparathyroidism

- history of athymia. ${ }^{1,28}$
Individuals with VCFS also display a distinct neuropsychological phenotype with deficits in executive functioning, visuospatial processing and working memory, which is a system for the shortterm storage and manipulation of information. ${ }^{13-17}$ In a recent study on DF (16) A1+ mice, which uses a microdeletion on the human chromosome 22 (22q11.2) model, it was suggested that impaired long-range synchrony of neural activity was one consequence of the $22 q 11$ deletion and may be a fundamental component of the pathophysiology underlying schizophrenia. ${ }^{18,19}$

Genetic research on schizophrenia now focuses on genomic copy number variations (CNVs). ${ }^{18}$ The $22 q 11.2,1 q 21.1$ and $15 q 13.3$ deletions are the only major genetic risk factors identified to date for schizophrenia, and for which clinical genetic assessment and testing are widely available. The relatively modest prevalence of these anomalies suggests to us that at present expensive genomic investigations are not warranted for all individuals with schizophrenia. ${ }^{18,19,20}$

Since 1997, 5 patients with VCFS-type schizophrenia have been identified in an ongoing collaborative study with Columbia University in the USA on schizophrenia in the Afrikaner population. Clinical data were collected from the Diagnostic Interview for Genetic Studies (DIGS) and DIGS Summary Report information. For each of the 5 patients who forms part of this review, we present a short case study describing each patient's unique features. The case studies are followed by short reviews of the literature on a particular feature. Where findings in the literature have a particular bearing on or reflect the unique features of any of these patients, this association is highlighted.

\section{Early deviant behaviour in VCFS Case 1}

John is the oldest of four siblings. His mother was healthy during pregnancy and he was born by forceps delivery. He was born with a VSD, which was later operated on, and defective valves in his urethra. At the age of 4 his frenulum had to be slit because of his indistinct speech. John has experienced problems with socialisation and has been afraid of the dark since the age of 2 . He had academic difficulties from the start of primary school, including problems of daydreaming and concentration that remained throughout his school career. He was diagnosed with attention deficit hyperactivity disorder and treated with methylphenidate. He also had periods of extreme sadness and anxiety.

At age 15 he reported that demons were causing him problems, and he experienced auditory hallucinations. After leaving school his functioning gradually deteriorated further and he was unable to finish his tertiary studies. At age 20 he became acutely psychotic and 
could not function in the open labour market. He had few friends and his working memory was found to be very poor. Features that point towards a poor prognosis included early onset of illness, obsessive-compulsive disorder (OCD), working memory deficits, and prominent negative symptoms. He fulfilled all the Bassett criteria for VCFS except history of hypocalcaemia and athymia.

Genetic testing confirmed the diagnosis of VCFS. A de novo mutation had caused the 22q11 deletion. Two of his siblings were born with craniostenosis, which has been associated with VCFS.

\section{Literature}

Early deviant behaviour in children who eventually develop schizophrenia has been reported consistently. ${ }^{2021}$ The domains of early deviant behaviour may be grouped into three clusters: a social functioning impairment cluster, a mood/anxiety cluster, and a cognitive impairment cluster. The social impairment cluster includes social isolation, aggression and extremely odd behaviour. The mood/anxiety cluster includes extreme fear and chronic sadness and the cognitive impairment cluster includes attention impairment and learning disability. All 5 of our patients whose cases are reviewed in this paper displayed early deviant behaviour.

Craniostenosis is a premature fusion of one or more of the cranial sutures. It may be seen as an isolated case or as part of the syndrome. In a study by McDonald-McGinn et al.,2 an association between craniostenosis and 22q11 DS was found. ${ }^{23-24}$ It was concluded that craniostenosis should be included in the diagnostic criteria of VCFS. Two of John's siblings had craniostenosis and should be further investigated for $22 q 11$ deletions. The results may have significant implications for providing genetic counselling to them.

\section{Dysmorphic features in VCFS \\ Case 2}

Peter is a 47-year-old man who lives in a chronic care facility. He is the youngest of 4 siblings, and was born with a congenital heart defect. He was socially isolated and his school progress was poor. He completed 2 years of military service and worked as a storeman for 10 years. At age 33, Peter became psychotic and suffered auditory hallucinations and paranoid delusions. After this episode he never worked again. He abused alcohol periodically and tried to commit suicide twice. Since the onset of psychosis he has not been able to function in society.

Peter has a poor prognosis as a result of his prominent negative features as well as the long duration of untreated psychosis. His diagnosis of VCFS was confirmed through genetic analysis, prompted by his meeting several of the Bassett criteria, including the facial dysmorphic features of a prominent nose, small chin, high hard palate, small malformed teeth and low-set ears, and a congenital heart defect. Other features were learning disability, long, tapering fingers, psychosis and nasal speech.

\section{Literature}

Dysmorphic features in VCFS include mild auricular abnormalities, hooded eyelids, a tubular-shaped nose, a broad nose tip, a small mouth, and slender and/or tapering fingers. Velopharyngeal insufficiency is one of the most consistent features of this syndrome and is usually confirmed in conjunction with a speech examination. Hypernasal resonance, weak pressure consonants and compensatory articulation are the typical speech symptoms related to velopharyngeal insufficiency. ${ }^{25}$ Other features are included in the Bassett criteria. ${ }^{1,19,25}$

Dysmorphic features were present in all 5 patients. Although Peter's dysmorphia was clearly observable, in some patients dysmorphia may require a careful examination before they will be recognised.

\section{Pathological laughter in VCFS \\ Case 3}

Julie is 27 years old and was initially referred because of an early onset of illness (she was 16 years old), as well as a treatment-resistant illness that suggested a poor prognosis. Julie is the youngest of 4 children and her siblings are all high achievers academically. She was born at 36 weeks' gestation and was kept in an incubator for 4 days after birth. Her developmental milestones were reached later than expected, and she received occupational therapy as a child. In primary school she had attention and concentration problems and was put in a special class. By age 16, children had reportedly teased her, she had become very anxious, and she was diagnosed with inappropriate laughter syndrome. Magnetic resonance imaging revealed a small lesion in the frontal area, which was thought to have been caused by birth trauma. She objectively hallucinated and suffered from the delusion that she was eating worms, snakes and frogs. She stopped attending school and did not participate in social activities.

Her parents report that at present Julie does not talk, her selfcare and functioning are very poor, and she finds changes in her environment or routine distressing. She suffers from fits of uncontrollable laughter that last up to 6 hours, and occur as often as 3 times a day and 3 times a week. During interviews she makes poor eye contact, talks softly to herself, and smiles inappropriately.

\section{Literature}

Pathological laughter is a syndrome of recurrent, episodic and involuntary laughter that occurs in the context of various neurological disorders. ${ }^{26-31}$ The exact mechanism by which this condition develops is currently unknown; however, there is general agreement that injury to pathways controlling emotional expression is involved. Emotional experience is the subjective feeling during an emotional event, whereas emotional expression is the objective behaviour that is experienced during such event. ${ }^{27,28}$ Both the experience and the expression of an emotion are dependent in part upon the cognitive appraisal of the emotional 
stimuli that are triggering them. ${ }^{27}$ Many psychiatric conditions lead to a problem with emotional experience (e.g. mood disorders), and many neurological disorders are associated with dysregulation of emotional expression. ${ }^{27,28}$ Pathological laughter and crying are often indistinguishable from normal laughter and crying. ${ }^{27}$ Since the outbursts may occur at socially inappropriate times, this problem causes social handicap and suffering. ${ }^{26,27,31}$

The neuro-anatomical basis for pathological laughter is poorly defined. Parvizi et al. propose that pathological laughter and crying (PLC) result from a disruption in the tracts originating from the primary motor cortex, which project topographically through the internal capsule and continue through the cerebral peduncles to the basis pontis and cerebellum (the cerebro-ponto-cerebellar pathways). ${ }^{27.28}$ They hypothesise that an insult to this pathway results in incomplete information being relayed to the cerebellar structures responsible for the execution of laughter and crying associated with the cognitive and situational context of a given stimulus.

Examples of neurological causes of PLC are pseudo-bulbar palsy (for example due to multiple sclerosis and motor neuron disease), gelastic seizures, Alzheimer's disease, multi-infarct dementia, Parkinson's disease and Down syndrome. ${ }^{272.39} \mathrm{PLC}$ responds to the selective serotonin reuptake inhibitors (SSRIs) that are used for mood disorders. ${ }^{28,30,31}$

Julie's MRI scan showed none of the structural abnormalities associated with neurological causes of PLC. The changes that were evident - namely atrophy and enlargement of the ventricles - are concordant with changes found in schizophrenia. A video electro-encephalogram revealed no abnormality. PLC has not been described in schizophrenia, but we hypothesise that the PLC may be attributed to VCFS and that the same pathways implicated in neurological causes of PLC may be affected in VCFS.

\section{OCD and working memory deficits in VCFS Case 4}

Haily is a 32-year-old divorced woman suffering from schizophrenia and obsessive-compulsive disorder (OCD). She is the oldest of 3 children, and her siblings are all good achievers. Haily's problems began at the age of 18, when she experienced visual hallucinations. After school she attended college and in her first year started hearing voices and thought people where talking about her. She found socialising very difficult and thought that people could read her mind. She had obsessive thoughts about her molesting other people. She attempted suicide impulsively at the age of 30.

Haily is currently a resident in an independent living unit. She functions at a low level and during her most recent interview had a flat affect and laughed inappropriately at times. A Letter-Number Span Test revealed very low scores, suggesting poor working memory.

\section{Literature}

OCD is estimated to occur in $13.2 \%$ of patients with schizophrenia, which is considerably higher than in the general population (2 $3 \%)^{32}$ The higher co-morbidity suggests the possibility of a common underlying pathogenesis for the diseases. There are structural and functional brain abnormalities common to schizophrenia and OCD, including common serotonin and dopamine neurotransmitter pathways. In recent studies it has been found that the clinical course of schizophrenia patients with OCD was more deteriorating than that of schizophrenia patients without OCD.

The schizophrenia-OCD co-morbidity is associated with poor treatment response, higher levels of negative symptoms, and more impairment in functioning. ${ }^{8}$ Studies by Gothelf et al. suggest that there is a strong association between VCFS and early-onset OCD. ${ }^{33}$ The COMT-gene is a candidate gene for schizophrenia because of its role in the breakdown of dopamine in the prefrontal cortex. CThe OMT high activity Val allele is associated with more obsessive compulsive symptoms in young patients with schizophrenia. The genetic contribution to the expression of OCD in patients with schizophrenia lends support to the validity of a putative schizoobsessive diagnostic entity. The current literature suggests that this co-morbid subgroup has a larger overall illness burden and these patients suffer worse outcomes than patients with schizophrenia without OCD. ${ }^{32.34}$

Regarding working memory in VCFS, measurements of brain activity in patients with schizophrenia suggest that functional connectivity between the frontal and temporal lobe, including the hippocampus, may be altered..$^{18}$

Working memory impairment is increasingly recognised as an important feature in the neuropsychology of schizophrenia. Dysfunction of working memory, a system for the short-term storage and manipulation of information, may relate to a number of core symptoms in schizophrenia. ${ }^{18}$ Working memory refers to short-term memory that is a flexible and dynamic memory resource where sensory information can be held briefly and manipulated. A study by Baddeley described working memory as a system for the temporary holding and manipulation of information during a range of neurocognitive tasks such as comprehension, learning and reasoning. This implies an active process rather than simply a static, temporary store. ${ }^{35}$

The dorsolateral prefrontal cortex (DLPC) is thought to play a major role in experience, thought, memory and emotion. The DLPC builds much of its circuitry during adolescence and childhood. Two cell types that play a major role in memory are the pyramidal cells and the chandelier cells. ${ }^{36}$ Multiple autopsy studies have shown that in people with schizophrenia, the pyramidal cells have smaller cell bodies and fewer dendritic spines. These cells control especially 
working memory. Chandelier cells can either exitate or inhibit pyramidal cells and it seems that during childhood these chandelier cells fail to cultivate pyramidal cells. This failure leads to the defect in thought, emotions and memory in schizophrenia. ${ }^{36}$

Cognitive problem deficits play a major role in the functional impairment in schizophrenia, especially the impairment in working memory. This is particularly the case in VCFS. ${ }^{35}$ Haily has severe functional impairment, which may be attributed to her working memory deficits as well as her co-morbid OCD. Her functioning has declined in the course of the 10 years since the diagnosis of schizophrenia.

\section{Later age of onset of schizophrenia \\ Case 5}

Jake presented with onset of schizophrenia at the age of 53 . He had passivity phenomena, auditory hallucinations, paranoid ideations, and grandiose delusions. He was born with a heart defect and his milestones were retarded. Jake has had difficulty socialising since a young age. In school he had concentration problems and he was moody and irritable. His final year at school was Grade 8. He was described as a 'loner' and his self-care was poor.

Jake married at the age of 35 even though he was homosexual. His wife came from a different cultural group and he lived with her in her cultural group. He was sent to prison for having sexual relationships with minors. He used alcohol socially, but never abused any substances. His mother had a psychiatric illness. Jake met all of the Bassett criteria except a history of athymia, hypocalcaemia and hypoparathyroidism. The diagnosis of VCFS was confirmed by fluorescent in situ hybridisation (FISH) analysis.

\section{Literature}

Schizophrenia typically has its onset during late adolescence or early adulthood whereas VCFS is associated with the even earlier onset of symptoms. ${ }^{1.12}$ Shprintzen et al. reported that some of their patients diagnosed with VCFS developed schizophrenia between the ages of 10 and $21{ }^{37}$ In adult-onset schizophrenia the incidence of VCFS is $2 \%$ and in child-onset schizophrenia (onset before the age of 13 ) it is $6 \%{ }^{1,8,12}$

The late onset of psychosis in VCFS has not previously been reported in the literature, as far as we could establish. Some patients with schizophrenia, however, develop symptoms only in middle age or old age. These patients with late-onset (older than 40) or very late-onset (older than 60) schizophrenia show similar characteristics to those with early-onset schizophrenia with regard to psychopathology, family history, cognitive deficits , nonspecific brain imaging abnormalities and treatment response.38 However, late- and very late-onset schizophrenia psychosis is more often associated with paranoid symptoms, less severe cognitive impairment and a need for lower dosages of antipsychotic medication. ${ }^{39.44}$ These patients also tend to have higher levels of education and relatively more of them are married. ${ }^{41-45}$ The proportion of patients with schizophrenia who present with lateonset schizophrenia has been estimated at $23 \%$. It is conceivable that factors associated with ageing such as retirement, financial difficulties, bereavement, the death of peers or physical disability may contribute to the precipitation of psychosis in later life. ${ }^{46}$

The mean age of onset of schizophrenia is 10 years later in women than in men. It is proposed that women may have protective factors early in life that result in a later onset of schizophrenia. ${ }^{47}$ It is also suggested that adults from migrant populations may be at higher risk. ${ }^{3.46}$ In Jake's case, the move to living in a different culture and the stress caused by the move may have precipitated the onset of psychosis.

\section{Conclusion}

In future, treatment of schizophrenia should ideally focus more preventing psychosis from developing in vulnerable individuals such as patients with VCFS. New developments in neuro-imaging of infants and children have the potential to improve our understanding of the critical periods in brain development. ${ }^{48}$ According to Gothelf et al., early recognition of 22q11DS patients at particularly high risk for psychosis will permit early intervention and decrease disability. ${ }^{2,33}$ Also, if patients are given a genetic diagnosis for their condition, they may be relieved of guilt or inappropriate blame for their behavioural manifestations of the condition. ${ }^{2}$

With regard to genetic counselling in VCFS it is important to note that there is a $50 \%$ chance of transmitting the deletion at each pregnancy, but the caveat is that the severity of the phenotype cannot be predicted. ${ }^{2}$ These individuals with VCFS have a $25 \%$ chance of developing psychosis. ${ }^{2}$ There is prenatal test available for the 22q11 deletion. 23,19,33 Patients with VCFS should be seen at regular intervals to screen for early deviant behaviour and prodromal symptoms, which may then be treated appropriately.

Detection of VCFS significantly changes genetic counselling and anticipatory care in comparison with the counselling and care given to other patients with schizophrenia. With respect to psychiatric diagnosis, these findings of copy number variations suggest that, as for the dementias, the schizophrenias may begin to yield to classification based on major causal factors. Comparable to an Alzheimer-type dementia associated with a beta-amyloid precursor protein (APP) gene mutation, a schizophrenia that is related to a 22q11.2 deletion (and perhaps a 1q21.1 or 15q13.3 deletion) may be considered a subtype with distinct management implications. ${ }^{19}$

In our findings, patients with VCFS can have varying clinical pictures and do not always have the same clinical presentation. Almost all of our patients suffered from an early onset of psychosis with poor prognostic features. All of them are functioning poorly - those who are not in hospital are living with their families. Early deviant behaviour as well as working memory deficits were prominent 
symptoms in all our patients. Nonetheless, VCFS can additionally present with many different faces - pathological laughter, family history of craniostenosis, and late onset of psychosis.

Clinical vigilance should be maintained for the presence of two or more of the Bassett criteria, which indicate a FISH analysis for confirmation of the VCFS diagnosis. It is necessary to do so because this disease has a different prognosis from that for other types of schizophrenia and appropriate genetic counselling is extremely important.

Acknowledgement. This publication was partially made possible by an incentive grant from the NRF to Professor J L Roos.

\section{References}

1. Wiehann GJ, Bosch GP, du Preez RR, Pretorius HW, Karayiorgou M, Roos JL. Assessment of the frequency of the 22q11 deletion in Afrikaner schizophrenic patients. Am J Med Genet B Neuropsychiatr Genet 2004;129B (1):20-22.

2. Basset A, Chow EW. 22q11 deletion syndrome: a genetic subtype of schizophrenia. Bio Psychiatry 1999;46(7):882-891.

3. Smith M. The year in human and medical genetics: highlights of 2007-2008. Ann NY Acad Sci 2009;151:1-21

4. Rockefeller University. Schizophrenia predisposition linked to two genes on chromosome 22 [press release on the Internet]. 19 March 2002. http://runews.rockefel!er.edu/index. php?page=engine\&id=14Q (accessed March 2010).

5. Bassett AS, Hodgkinson K, Chow EW, et al. 22q11 deletion syndrome in adults with schizophrenia. Am J Med Genet 1998;81:328-337.

6. Scambier PJ. The 22q11 deletion syndromes. Hum Mol Genet 2000;9(16):2421-2426

7. Murphy $K G$, Jones $L A$, Owen $M J$. High rates of schizophrenia in adults with velo-cardiofacial syndrome. Arch Gen Psychiatry 1999;56(10):940-945.

8. Roos $J$, Pretorius HW, Karayiorgou M. Clinical characteristics of an Afrikaner founder population recruited for a schizophrenia genetic study. Ann NY Acad Sci 2009;1 151: 85-101.

9. Liu H, Abecasis GR, Heath SC, et al. Genetic variation in the 22 q11 locus and susceptibility to schizophrenia. Proc Nat Acad Sci 2002;99(26):6859-6864

10. Chackravarti A. A compelling genetic hypothesis for a complex disease: PRODH2/DGCR6 variation leads to schizophrenia susceptibility. Proc Natl Acad Sci USA 2002;99(8):4755 4756.

11. Usiskin SI, Nicolson R, Krasnewich DM, et al. Velocardiofacial syndrome in childhood-onset schizophrenia. J Am Acad Child Adolesc Psychiatry 1999;38(12):1536-1543.

12. Liu H, Heath SC, Sobin C, et al. Genetic variation at the 22q11 PRODH2/DGCR6 locus presents an unusual pattern and increases susceptibility to schizophrenia. Proc Natl Acad Sci USA 2002;99(6):3717-3722.

13. Kates WR, Krauss BR, AbdulSabur N, et al. The neural correlates of non-spatial working memory in velocardiofacial syndrome (22q11.2 deletion syndrome). Neuropsychologia 2007:45(12):2863-2873.

14. Lewandowski KE, Shashi V, Berry PM, Kwapil TR. Schizophrenic-like neurocognitive deficits in children and adolescents with 22q11 deletion syndrome. Am J Med Genet B Neurospychiatr Genet 2007;144B(1):27-36.

15. Sobin C, Kiley-Brabeck K, Daniels S, et al. Neuropsychological characteristics of children with the 22q11 deletion syndrome: a descriptive analysis. Child Neuropsychol 2005;11(1):39-53.

16. Meyer-Lindberg AS, Olsen RK, Kohn PD, et al. Regionally specific disturbance of dorsolateral prefrontal hippocampal functional connectivity in schizophrenia. Arch Gen Psychiatry 2005;62(4):379-386.

17. Karayiorgou M, Simon TJ, Gogos JA. 22q11.2microdeletions: linking DNA structural variation to brain dysfunction and schizophrenia. Nature 2010;11:402-416.

18. Sigurdsson T, Stark KL,Karayiourgou M, Gogos JA, Gordon JA. Impaired hippocampalprefrontal synchrony in a genetic mouse model of schizophrenia. Nature 2010; 464:736-737.

19. Basset AS, Scherer SW, Brzustowicz LM. Copy number variations in schizophrenia: critical review and new perspectives on concepts of genetics and disease. Am J Psychiatry 2010;167:899-914
20. Sobin C, Roos JL, Pretorius H, Lundy LS, Karayiorgou M. A comparison study of early nonpsychotic behavior in Afrikaner and US patients with schizophrenia or schizoaffective disorder. Psychol Res 2003;117:113-125.

21. Scholtz MC, Janse van Rensburg M, Roos JL, et al. Early non-psychotic deviant behavior as an endophenotypic marker in bipolar disorder, schizoaffective disorder and schizophrenia. South African Psychiatry Review 2002:8:153-159.

22. McDonald-McGinn DM, Gripp KW, Kirscner E, et al. Craniostenosis: Another feature of the 22q11Deletion Syndrome. Am J Med Genet A 2005;136:358-362.

23. Ryan AK, Goodship JA, Wilson DI, et al. Spectrum of clinical features associated with interstitial chromosome 22q11 deletions: a European collaborative study. J Med Genet 1997;34:798-804.

24. Dean JC, De Silva DC, Reardon W. Craniostenosis and chromosome 22q11 deletion. J Med Genet 1998;35:346.

25. Oskarsdottir S, Persson C, Eriksson BO, Fasth A. Presenting phenotype in 100 children with the 22q11 deletion syndrome. Eur J Pediatr 2005;164:146-153.

26. Okuda DT, Chyung ASC, Chin CT, Waubant W. Acute pathological laughter. Mov Disord 2005;20:1389-1390.

27. Parvizi J, Coburn KL, Shillcutt SD, et al. Neuroanatomy of pathological laughing and crying: a report of the American Neuropsychiatry Association Committee on Research. J Neuropsychiatry Clin Neurosci 2009;21(1):75-87.

28. Parvizi J, Arciniegas DB, Bernardini G, et al. Diagnosis and management of pathological laughter and crying. Mayo Clin Proc 2006;81(11):1482-1486.

29. Nadkarni TD, Goel A. Trochlear nerve neurinoma presenting as pathological laughter. $\mathrm{Br}\rfloor$ Neurosurgery 1999;13(2):212-213.

30. Mendez MF, Nakawatase TV, Brown CV. Involuntary laughter and inappropriate hilarity. J Neuropsychiatry Clin Neurosci 1999;1 1(2):253-258.

31. Møller M, Andersen G. Inhibition of selective noradrenergic reuptake as treatment of pathological laughter. J Clin Psychopharmacol 2007;27(1):108-110.

32. Seedat F, Roos JL, Pretorius HW, Karayiorgou M, Nel B. Prevalence and clinical characteristics of obsessive-compulsive disorder and obsessive compulsive symptoms in Afrikaner schizophrenia and schizoaffective disorder patients. African Journal of Psychiatry 2007;10(4):219-224.

33. Gothelf $D$, Presburger $G$, Zohar AH, et al. Obsessive-compulsive disorder in patients with velocardiofacial (22q11 deletion) syndrome. Am J Med Genet 2004;1268(1):99-105.

34. Bottas A. Comorbidity: schizophrenia with obsessive compulsive disorder. Psychiatric Times [serial on the Internet]. 15 April 2009. http://www.psychiatrictimes.eom/display/ articie/10168/1402540 (accessed March 2010).

35. Baddeley A. Working memory. Science 1992;255:556-559.

36. Dobbs D. The making of a troubled mind. Nature 2010;468:154-157.

37. Shprintzen RJ, Goldberg R, Golding-Kushner KJ, Marion RW. Late onset psychosis in the velo cardio facial syndrome. Am J Med Genet 1992;42:141-142.

38. Cohen $C L$, Vahia I, Reyes $P$, et al. Focus on geriatric psychiatry: Schizophrenia in later life: clinical symptoms and social wellbeing. Psychiatr Serv 2008;59(3):232-234.

39. Psarros C, Theleritis CG, Paparrigopoulos TJ, Politis AM, Papadimitriou GN. Amisulpiride for the treatment of very late onset schizophrenia like psychosis. Int J Geriatr Psychiatry 2009;24:518-522.

40. Girard C, Simard M. Clinical characteristics of late and very late onset first psychotic episode in psychiatric inpatients. Am J Geriatr Psychiatry 2008;16:478-487.

41. Howard R, Rabins PV, Seeman MV, Jeste DV. Late onset and very late onset schizophrenialike psychosis: An international consensus. Am J Psychiatry 2000;157:172-178.

42. Schurhoff F, Golmard JL, Szoke A, et al. Admixture analysis of age at onset in schizophrenia. Schizophr Res 2004;71:35-41.

43. Alici-Evcimen $Y$, Ertan T, Eker E. Case series with late onset psychosis hospitalized in a geriatric psychiatry unit in Turkey. Int Psychogeriatrics.2003;15:69-72.

44. Mazeb D, Zemisblani C, Aizenberg D, Barak Y. Patients with very late onset schizophrenia like psychosis. Am J Geriatr Psychiatry 2005;13:417-419.

45. Sato T, Bottlender R, Schroter A, Moller HJ. Psychopathology of early onset versus late onset schizophrenia revisited: an observation of 473 neuroleptic naïve patients before and after first admission treatments. Schizophr Res 2004;67:175-183.

46. Rabins PV, Lavrisha M. Long term follow-up and phenemologic differences distinguish among late onset schizophrenia, late life depression and progressive dementia. Am J Geriatr Psychiatry 2003;11:589-594.

47. Mitter P, Reeves S, Romero-Rubiales F, et al. Migrant status, age, gender and social isolation in very late onset schizophrenia like psychosis. Int J Geriatr Psychiatry 2005;20:1046-1051.

48. Ross RG. Neuroimaging the infant: the application of modern neurobiological methods to the neurodevelopmental hypothesis of schizophrenia. Am J Psychiatry 2010;167:1017 1018 